\title{
Identification of candidate antigens from adult stages of Toxocara canis for the serodiagnosis of human toxocariasis
}

\author{
Patrícia Longuinhos Peixoto', Evaldo Nascimento², Guilherme Grossi Lopes Cançado1, \\ Rodrigo Rodrigues Cambraia de Miranda', Regina Lunardi Rocha ${ }^{3}$, \\ Ricardo Nascimento Araújo ${ }^{1}$, Ricardo Toshio Fujiwara ${ }^{1 /+}$
}

\author{
${ }^{1}$ Departamento de Parasitologia, Instituto de Ciências Biológicas \\ ${ }^{3}$ Departamento de Pediatria, Faculdade de Medicina, Universidade Federal de Minas Gerais, Belo Horizonte, MG, Brasil \\ ${ }^{2}$ Centro de Ensino e Pesquisa, Hospital Santa Casa de Belo Horizonte, Belo Horizonte, MG, Brasil
}

In the present work, we identified adult Toxocara canis antigens through sodium dodecyl sulfate-polyacrylamide gel electrophoresis for potential use in human toxocariasis immunodiagnosis. The sensitivity and specificity of several semi-purified antigens, as well as their cross-reactivity with other parasitic infections, were assessed by $I g M$ and IgG-enzime linked immunosorbent assay. Whilst we found that the crude extract of the parasite presented limited sensitivity, specificity and high cross-reactivity against other parasites, we identified 42, 58, 68 and 97$k D a$ semi-purified antigens as the most promising candidates for immunodiagnosis. Moreover, the 58 and $68-k D a$ antigens presented the lowest IgM cross-reactivity. When tested as a combination, a mixture of the 58 and $68-k D a$ antigens presented $100 \%$ sensitivity and specificity, as well as minor cross-reactivity. Although the combination of the 42, 58, 68 and 97- $k D a$ antigens presented 100\% sensitivity at a dilution of 1:40, the low specificity and high cross-reactivity observed suggested a limited use for diagnostic purposes. Our data suggested that the 58 and 68 $k D a$ antigens might be most suitable for the immunodiagnosis of human toxocariasis.

Key words: Toxocara - ELISA - visceral larva migrans

Human toxocariasis is a worldwide helminthic zoonosis caused by Toxocara canis and Toxocara cati, the common ascarids of dogs and cats, respectively (Schantz \& Glickman 1983, Despommier 2003). Humans are infected by accidental ingestion of embryonated Toxocara eggs. The infective larvae hatch in the duodenum, but the juvenile parasites fail to develop to mature adult worms (Despommier 2003). Instead, they migrate through the somatic organs for months or up to several years, causing three recognizable syndromes: visceral larva migrans, ocular toxocariasis and covert toxocariasis (Glickman \& Schantz 1981, Taylor et al. 1988, Jacob et al. 1994). Children playing in areas contaminated with dog faeces, as well as people with intimate contact with dogs, are subject to a higher risk of infection (Habluetzel et al. 2003, Wolfe \& Wright 2003, Espinoza et al. 2008). In the United States, human toxocariasis is the most common human parasitic worm infection and affects $13.9 \%$ of the population, particularly minorities groups such as nonHispanic blacks and Latin Americans immigrants (Jones et al. 2008, Hotez \& Wilkins 2009). In the developing countries, this scenario is probably worse and the crossreactivity with antibodies from other endemic helminth infections is a limitation for the development of an accu-

Financial support: CAPES, CNPq, FAPEMIG

RNA and RTF contributed equally to this work.

+ Corresponding author: fujiwara@icb.ufmg.br

Received 23 August 2010

Accepted 16 December 2010 rate serodiagnostic test [enzime linked immunosorbent assay (ELISA)] using the current available unfractionated excretory-secretory (ES) antigens from the T. canis second-stage larvae (TES Ag) (Smith et al. 2009).

The high prevalence of Toxocara spp and the non-periodic deworming of domestic pets (dogs and cats) result in mass contamination of the ground. Previous reports demonstrated that between $0-93 \%$ of dogs are infected (Glickman \& Schantz 1981) and between $15-78 \%$ of soil is contaminated (Gillespie 1988, Magnaval et al. 2001). These issues are associated with the absence of hand-washing habits that prevent faecal-oral contact and undoubtedly contribute to the high prevalence of human toxocariasis in children who live in urban and rural poor hygiene areas (Jones et al. 1980, Marmor et al. 1987). Moreover, human toxocariasis is not a communicable disease in the majority of countries and the incidence of human infection remains unknown and probably underestimated.

The diagnosis of human toxocariasis depends on clinical and serological data because detecting Toxocara larvae by biopsy is difficult (Yamasaki et al. 2000, Roldán et al. 2006). Since the development of the ELISA using ES antigens from TES Ag described by de Savigny et al. (1979) and the subsequent western-blot method using the same antigen by Magnaval et al. (1991), a general agreement has existed regarding the value of the TES Ag for the immunodiagnosis of human toxocariasis (Glickman et al. 1978, Lynch et al. 1988, Jacquier et al. 1991, Gillespie et al. 1993, Roldán et al. 2006). However, the production of TES Ag is difficult and expensive and the process requires expertly trained scientists and technicians. The possibility of using $T$. canis adult antigenic 
extracts, which are by far easier to produce, would therefore facilitate the execution of diagnostic methods. The fractionation and isolation of antigens decrease the chance of cross-reactivity with other parasitic infections and could improve the current serodiagnostic tests.

The aim of the present study was to identify specific antigens from the adult stages of $T$. canis that may be useful for the serodiagnosis of human toxocariasis. To improve the immunodiagnosis of the human visceral larva migrans, we determined the sensitivity and specificity of several semi-purified antigens, as well as their cross-reactivity with other parasitic infections.

\section{SUBJECTS, MATERIALS AND METHODS}

Isolation of parasites - Upon necropsy of 47 young dogs (aged from 1-4 months) that were naturally infected with Toxocara, we harvested 200 male and female $T$. canis adult worms. This was performed at the Centre for Control of Zoonosis, Belo Horizonte, Minas Gerais (MG), Brazil. The parasites were repeatedly washed with phosphate buffered saline (PBS) at pH 7.2 and cryopreserved at $-20^{\circ} \mathrm{C}$.

Antigen preparation - The crude extracts of adult $T$. canis were obtained by maceration of the worms using a tissue grinder in the presence of PBS at pH $7.2(8.1 \mathrm{mM}$ $\mathrm{Na}_{2} \mathrm{PO}_{4}, 1.5 \mathrm{mM} \mathrm{KH} \mathrm{PO}_{4}, 136 \mathrm{mM} \mathrm{NaCl}$ ) and protease inhibitors $(1 \mathrm{mM}$ tosyl-L-lysine chloromethyl ketone, $100 \mu \mathrm{M}$ iodoacetamide and $10 \mu \mathrm{g} / \mathrm{mL}$ Pepstatin A). The parasite extract was centrifuged for $1 \mathrm{~h}$ at 20,800 $\mathrm{g}$ and $8^{\circ} \mathrm{C}$. The supernatant was aliquoted and cryopreserved at $-20^{\circ} \mathrm{C}$ until needed. The antigen concentration was determined using the method described by Lowry et al. (1951).

Sodium dodecyl sulphate-polyacrylamide gel electrophoresis (SDS-PAGE) - The crude parasite extract $(150 \mu \mathrm{L}$ of antigen at a concentration of $3.5 \mu \mathrm{g} / \mu \mathrm{L}$ mixed with $150 \mu \mathrm{L}$ of sample buffer composed of $50 \mathrm{mM}$ Tris- $\mathrm{HCl}$ at pH $6.8,2 \%$ SDS, $10 \%$ glycerol, $1 \% \beta$-mercaptoethanol, $12.5 \mathrm{mM}$ ethylenediamine tetraacetic acid and $0.02 \%$ bromophenol blue) was boiled for $5 \mathrm{~min}$ and then separated on a $4.5-12.5 \%$ gradient SDS-PAGE at a constant current of $100 \mathrm{~V}$ for $4 \mathrm{~h}$. The following molecular weight standards (GIBCO/BRL, MD, USA) were included: (i) 205-kDa myosin (H chain), (ii) 116-kDa $\beta$-galactosidase, (iii) 97.4-kDa phosphorilase b, (iv) 66-kDa bovine albumin serum and (v) 29-kDa carbonic anidrase. The individual antigen bands were then visualized by Coomassie Brilliant Blue (Sigma, USA) staining.

Electroelution - The gel bands with each separated antigen were excised ( $3 \times 3 \mathrm{~mm})$, placed onto dialysis membranes containing elution buffer $(125 \mathrm{mM}$ Tris/HCI at $\mathrm{pH}$ 8.5 and $0.1 \%$ SDS) and submitted to a constant current of $100 \mathrm{~V}$ for $1 \mathrm{~h}$. The antigens were dialysed in PBS at $\mathrm{pH}$ 7.2 for $72 \mathrm{~h}$ and then concentrated to a final volume of $500 \mu \mathrm{L}$. The purification of the antigens was confirmed by SDS-PAGE stained with Coomassie Brilliant Blue.

Human serum - Serum samples $(\mathrm{n}=20)$ were obtained from patients with confirmed clinical diagnosis of toxocariasis, as determined by signs of visceral involvement in association with hypergammaglobulinemia, leu- kocytosis, eosinophilia and negative faecal parasitological exams (Kato-Katz and Baermann-Moraes). For the analysis of cross-reactions, patients with the following parasitic infections were included: schistosomiasis (n $=20)$, neurocysticercosis $(\mathrm{n}=20)$ and Chagas disease $(n=20)$. Schistosomiasis was confirmed by repeated parasitological tests, neurocysticercosis by computerized tomography and Chagas disease by clinical and serological tests (Iudici Neto et al. 2007). The sera were obtained after informed consent at the Clinical Hospital of the Federal University of Minas Gerais (UFMG). As negative controls, the sera from healthy non-exposed donors $(n=20)$ were collected at the Palo Alto Medical Foundation (Palo Alto, USA).

Absorption with Ascaris suum extract - A. suum were obtained from naturally infected pigs in the region of Montes Claros, MG, Brazil (Iudici Neto et al. 2007). Fifty microliters of each serum sample was absorbed with $50 \mu \mathrm{L}$ of A. suum antigenic extract (prepared as mentioned) at a concentration of $5.0 \mu \mathrm{g} / \mathrm{mL}$ for $24 \mathrm{~h}$. This procedure was performed to remove the non-specific antibodies that might cross-react with the semi-purified T. canis antigens, as extensively described by several authors (Glickman et al. 1978, de Savigny et al. 1979, Roldán et al. 2006).

ELISA - One hundred microliters of semi-purified $(30 \mu \mathrm{g} / \mathrm{mL})$ or crude $(50 \mu \mathrm{g} / \mathrm{mL})$ T. canis antigens diluted in carbonate buffer $0.1 \mathrm{M}\left(1.59 \mathrm{~g} / \mathrm{L} \mathrm{NaCO}_{3}, 2.93 \mathrm{~g} / \mathrm{L}\right.$ $\mathrm{NaHCO}_{3}, 0.2 \mathrm{~g} / \mathrm{L} \mathrm{NaN}$ ) at $\mathrm{pH} 9.6$ were coated onto 96well polystyrene microplates (Interlab, São Paulo, Brazil) and incubated for $18 \mathrm{~h}$ at $4^{\circ} \mathrm{C}$. The sera were added at a dilution of 1:40 and two-fold dilutions thereafter until a dilution of 1:320 and incubated for $90 \mathrm{~min}$ at room temperature (RT). After incubation, the wells were washed five times with $0.5 \%$ Tween 20 in PBS and $100 \mu \mathrm{L}$ of either peroxidase-conjugated anti-human IgG (Cappel, USA) at a 1:5,000 dilution or peroxidase-conjugated anti-human IgM (Cappel, USA) at a 1:2,500 dilution was then added for incubation for $60 \mathrm{~min}$ at RT. After another round of washing, the reaction was developed by adding $100 \mu \mathrm{L}$ of phosphate-citrate buffer solution (46 mM citric acid, $100 \mathrm{mM} \mathrm{Na} 2 \mathrm{PO}_{4}$ ) containing $0.03 \%$ orthophenylenediamine (Sigma) and $0.04 \%$ hydrogen peroxide (Sigma) for $20 \mathrm{~min}$ at RT. Finally, $25 \mu \mathrm{L}$ of $4 \mathrm{~N}$ $\mathrm{H}_{2} \mathrm{SO}_{4}$ was added to each well to stop the reaction. The absorbance was read on an automatic ELISA microplate reader (BioRad, USA) at the wavelength of $492 \mathrm{~nm}$.

A positive-negative cut-off value was determined by calculating the mean values of absorbance from the negative sera plus three times the standard deviation. The absorbance values above the cut-off were considered positive, whilst values that were below were considered negative. The cut-off values were determined using all the negative sera and were adjusted for each plate by including internal calibrators. Multiple combinations of the semi-purified antigens (molecular weights $42 \mathrm{kDa}$, $58 \mathrm{kDa}, 68 \mathrm{kDa}$ and $97 \mathrm{kDa}$ ) were made at the final concentration of $30 \mu \mathrm{g} / \mathrm{mL}$. The sensitivity and specificity calculations were performed according to Iudici Neto et al. (2007) and Buck and Gart (1966). 
Ethics - All experiments were conducted in accordance with an approved protocol previously submitted to the UFMG Ethics Committee for Human Research (ETIC 223/02).

\section{RESULTS}

To develop a reliable serological test for the diagnosis of human toxocariasis, the sensitivity and specificity of a crude adult worm antigen were first tested to allow further comparisons with semi-purified antigens. Using the crude antigen and sera from Toxocara-infected donors, we developed an ELISA that discriminated between patients, thus presenting a $100 \%$ sensitivity for both IgM and IgG antibodies at a dilution 1:40 (Fig. 1A, B). The sensitivity was reduced when the serum titration reached values as low as $10 \%$ for $\operatorname{IgM}$ and $\mathrm{IgG}$ at a dilution of 1:320. Conversely, the non-specific reactions to negative sera for both IgM and IgG increased as the serum was
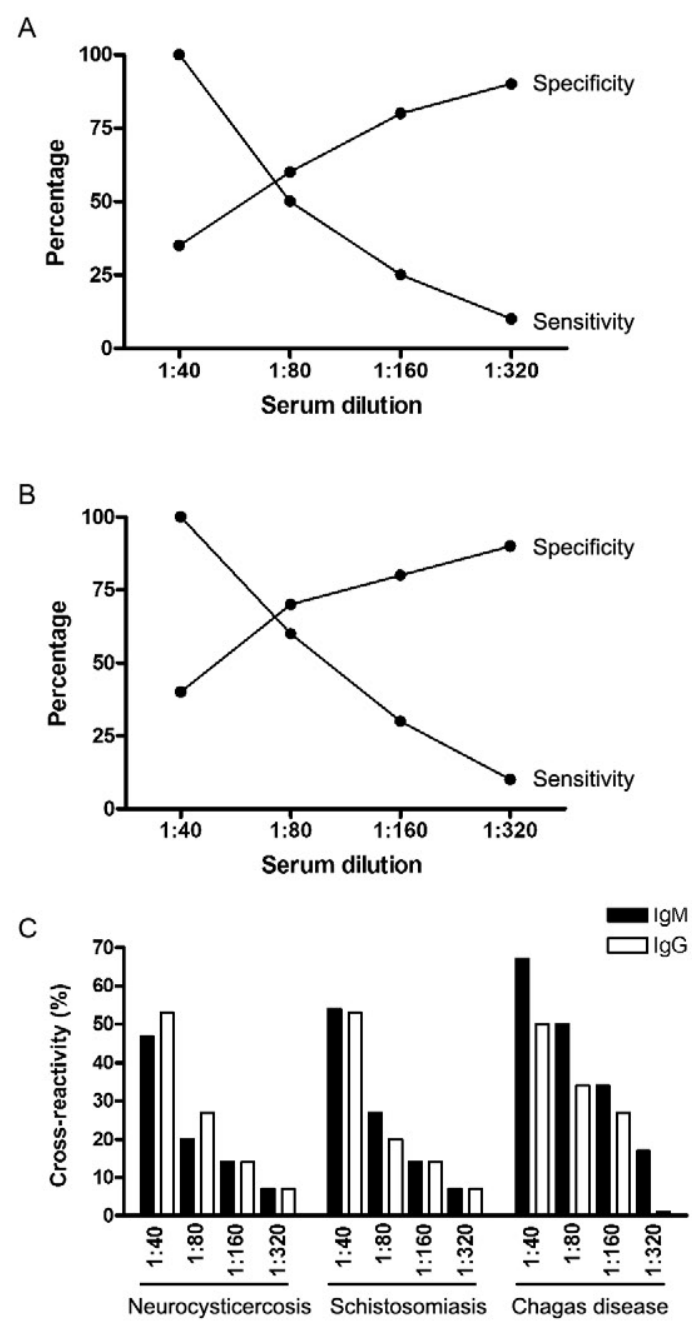

Fig. 1: sensitivity and specificity of crude adult Toxocara canis antigen at different serum dilutions determined by enzime linked immunosorbent assay (ELISA). A: sensitivity and specificity by IgMELISA; B: sensitivity and specificity by IgG-ELISA; C: percentage of serum cross-reactivity in IgM and IgG-ELISA against serum samples from individuals infected with other parasitic infections. diluted. The serum dilution that presented the best combination of sensitivity and specificity for $\operatorname{IgM}$ and $\operatorname{IgG}$ was 1:80. However, at this dilution, the cross-reactivity observed with other parasitic infections was as high as $20-50 \%$ (Fig. 1C).

Considering the limited sensitivity, specificity and high cross-reactivity presented by the crude Toxocara antigen, we performed the identification, separation and testing of the semi-purified antigens. The electrophoresis of the crude antigen identified major antigen bands with estimated molecular weights of 210, 120, 97, 95, 68, $58,42,40$ and $30 \mathrm{kDa}$ (Fig. 2). The antigens with molecular weights of 42, 58, 68 and $97 \mathrm{kDa}$ demonstrated considerable sensitivity and specificity (higher than 90\% for both) for IgM and IgG isotypes at the dilution of 1:40 as shown in Fig. 3. Although the specificity remained elevated at all dilutions, the sensitivity waned according to the sera titration (Fig. 3). When the sera from patients with other parasitic infections were tested, the same antigens presented variable cross-reactivity (Table I). We noted that the IgM ELISA presented overall lower values of cross-reactivity when compared with those found for $\mathrm{IgG}$. With regard to the IgG ELISA, only the $68-\mathrm{kDa}$ antigen presented moderate (up to $17 \%$ ) cross-reactivity at the same dilution (Table I). Among all the antigens, the 58 and $68-\mathrm{kDa}$ antigens presented the lowest $\mathrm{IgM}$ and $\mathrm{IgG}$ reactivity against other parasites even at a dilution of 1:40 and we then tested these antigens together in combination. The other semi-purified fractions of $T$. canis adult antigens were also tested and these presented unsatisfactory sensitivity and/or specificity results (Table II).

Finally, to verify whether the combination of different semi-purified antigens might improve the immunodiagnostics, we tested the following two mixtures: the 58 and $68-\mathrm{kDa}$ antigens and the 42,58,68 and $97-\mathrm{kDa}$ antigens (Fig. 4). The 58 and $68-\mathrm{kDa}$ combination presented $100 \%$ sensitivity and specificity, as well as minor cross-reactivity at a dilution of 1:40 for both $\operatorname{IgM}$ and IgG. Although the specificity and cross-reactivity remained stable along with the serum titration, the sensitivity was considerably reduced from the dilution of 1:80 onwards (Fig. 4A, B). Although 42, 58, 68 and 97-kDa antigen combination presented $100 \%$ sensitivity

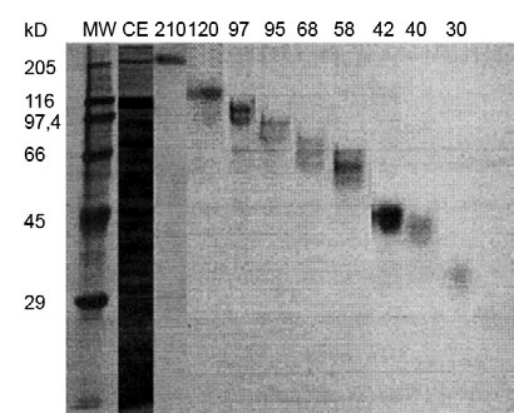

Fig. 2: sodium dodecyl sulphate-polyacrylamide gel electrophoresis analysis of crude adult Toxocara canis antigen and semi-purified antigens by electroelution. Lane 1: molecular weight (MW); 2: crude adult extract of $T$. canis (CE); 3-11: semi-purified antigens. 

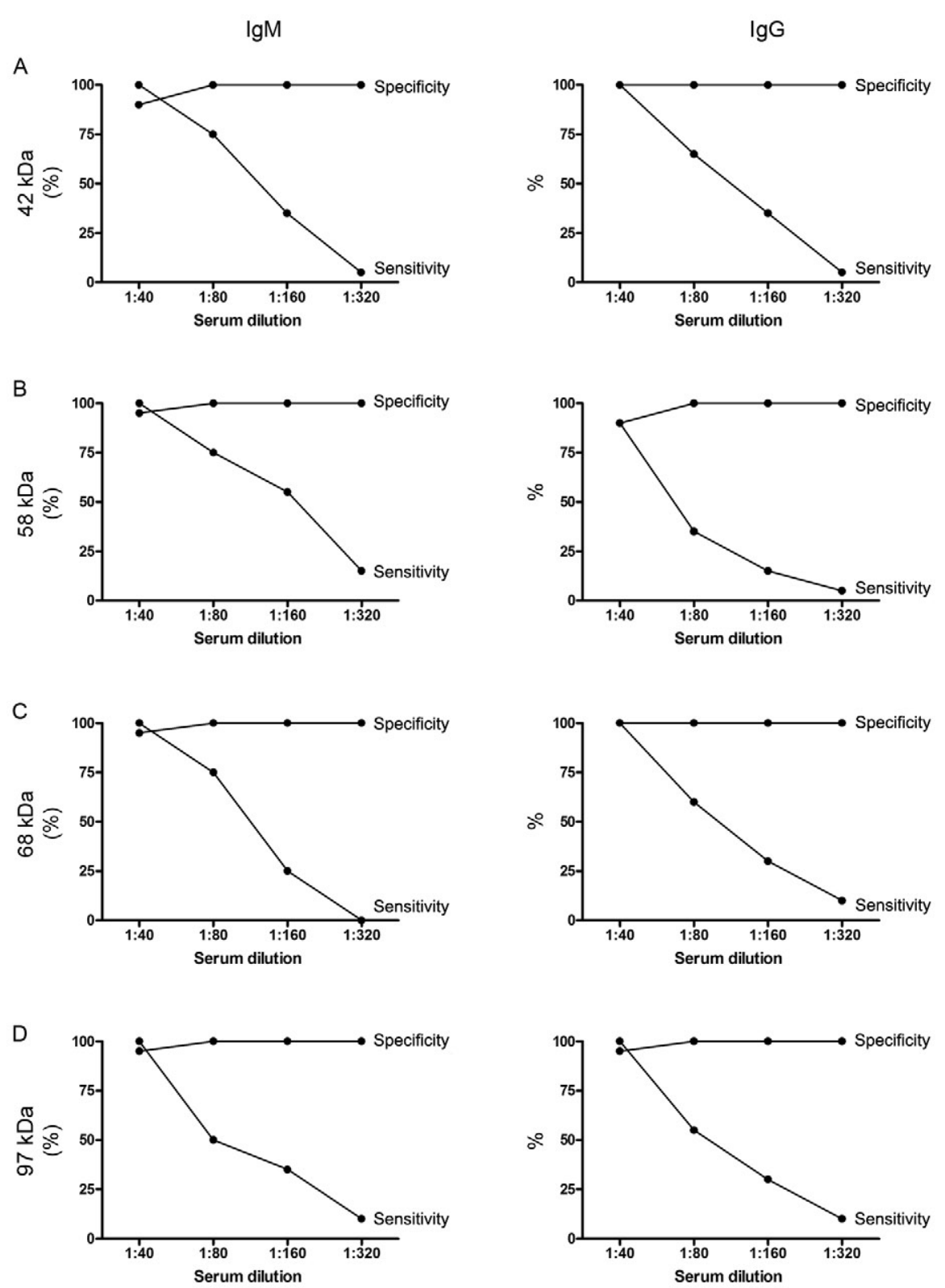

Fig. 3: sensitivity and specificity of semi-purified Toxocara canis antigens at different serum dilutions determined by IgM (left panel) or IgGenzime linked immunosorbent assay (ELISA) (right panel). ELISAs were performed using the following antigens: A: $42 \mathrm{kDa} ; \mathrm{B}: 58 \mathrm{kDa}$; C: 68 $\mathrm{kDa}$; D: $97 \mathrm{kDa}$.

at a dilution of 1:40, the low specificity and high crossreactivity observed suggested the limited use of this mixture for the diagnosis of human toxocariasis.

\section{DISCUSSION}

Human toxocariasis is one of the most common zoonotic helminthiases in temperate and tropical countries and occurs periodically in urban and rural areas as a consequence of accidental infection (Jones et al. 1980, 2008, Schantz 1989, Gillespie et al. 1993). In Brazil, seroprevalence surveys have found that up to $21.8 \%$ of children under the age of 12 are infected (De Andrade Lima Coelho et al. 2005). In fact, the high prevalence rates, which may be related to intestinal polyparasitism, raw food intake, intimate contact with dogs at home and soil contamination, have been reported in several countries (Giacometti et al. 2000, Chiodo et al. 2006, Gawor et al. 2008). Therefore, the development of a reliable $T$. canis-specific ELISA would be recommended not only for routine diagnosis, but also for seroepidemiologic surveys of toxocariasis in humans.

The diagnosis of toxocariasis is based on clinical and serological data because of the difficulty in detecting the larvae in tissues (e.g., biopsy). The test currently employed for the serodiagnosis of toxocariasis is an ELISA that uses TES Ag (de Savigny et al. 1979, Jacquier et al. 


\section{TABLE I}

Reactivity of semi-purified Toxocara sp. antigens against serum samples from patients infected with other parasites

\begin{tabular}{|c|c|c|c|c|c|c|c|c|c|}
\hline \multirow{2}{*}{$\begin{array}{l}\text { Antigens } \\
(\mathrm{kDa})\end{array}$} & \multirow{2}{*}{$\begin{array}{c}\text { Serum } \\
\text { reactivity (\%) }\end{array}$} & \multicolumn{4}{|c|}{ Titers 1: IgM } & \multicolumn{4}{|c|}{ Titers 1: IgG } \\
\hline & & 40 & 80 & $160 ?$ & 320 & 40 & 80 & 160 & 320 \\
\hline \multirow[t]{3}{*}{42} & Neurocysticercosis & 0 & 0 & 0 & 0 & 34 & 7 & 0 & 0 \\
\hline & Schistosomiasis & 27 & 7 & 0 & 0 & 34 & 14 & 7 & 0 \\
\hline & Chagas disease & 17 & 0 & 0 & 0 & 0 & 0 & 0 & 0 \\
\hline \multirow[t]{3}{*}{58} & Neurocysticercosis & 7 & 0 & 0 & 0 & 34 & 7 & 0 & 0 \\
\hline & Schistosomiasis & 0 & 0 & 0 & 0 & 27 & 7 & 0 & 0 \\
\hline & Chagas disease & 0 & 0 & 0 & 0 & 0 & 0 & 0 & 0 \\
\hline \multirow[t]{3}{*}{68} & Neurocysticercosis & 0 & 0 & 0 & 0 & 7 & 0 & 0 & 0 \\
\hline & Schistosomiasis & 7 & 0 & 0 & 0 & 0 & 0 & 0 & 0 \\
\hline & Chagas disease & 0 & 0 & 0 & 0 & 17 & 0 & 0 & 0 \\
\hline \multirow[t]{3}{*}{97} & Neurocysticercosis & 7 & 0 & 0 & 0 & 14 & 0 & 0 & 0 \\
\hline & Schistosomiasis & 40 & 14 & 7 & 0 & 27 & 7 & 0 & 0 \\
\hline & Chagas disease & 50 & 34 & 17 & 0 & 67 & 34 & 17 & 0 \\
\hline
\end{tabular}

\section{TABLE II}

Sensitivity and specificity of semi-purified Toxocara sp. antigens against serum samples from visceral larva migrans infected patients and healthy donors

\begin{tabular}{|c|c|c|c|c|c|c|c|c|c|}
\hline \multirow{2}{*}{$\begin{array}{l}\text { Antigens } \\
(\mathrm{kDa})\end{array}$} & \multirow[b]{2}{*}{ Rates (\%) } & \multicolumn{4}{|c|}{ Titers 1: IgM } & \multicolumn{4}{|c|}{ Titers 1: IgG } \\
\hline & & 40 & 80 & 160 & 320 & 40 & 80 & 160 & 320 \\
\hline \multirow[t]{2}{*}{30} & Sensitivity & 80 & 20 & 10 & 5 & 25 & 15 & 10 & 5 \\
\hline & Specificity & 80 & 95 & 100 & 100 & 70 & 100 & 100 & 100 \\
\hline \multirow[t]{2}{*}{95} & Sensitivity & 70 & 25 & 20 & 5 & 70 & 40 & 30 & 10 \\
\hline & Specificity & 75 & 95 & 100 & 100 & 75 & 90 & 95 & 100 \\
\hline \multirow[t]{2}{*}{120} & Sensitivity & 65 & 15 & 5 & 0 & 60 & 5 & 0 & 0 \\
\hline & Specificity & 50 & 75 & 80 & 90 & 25 & 55 & 60 & 75 \\
\hline \multirow[t]{2}{*}{210} & Sensitivity & 100 & 80 & 55 & 30 & 80 & 40 & 20 & 20 \\
\hline & Specificity & 45 & 80 & 95 & 100 & 80 & 80 & 85 & 90 \\
\hline
\end{tabular}

the $40 \mathrm{kDa}$ antigen had no reactivity for $\operatorname{IgG}$ or IgM.

1991). Although the TES-ELISA has reasonable sensitivity and specificity, this assay may yield false-positive results because of cross-reactivity with other parasitic infections. In addition, the TES-ELISA has low concordance rates between different batches due to variations in the TES preparations (Smith \& Noordin 2006) and the in vitro culture of T. canis larvae and the harvesting of
TES antigens are laborious and time consuming (Speiser \& Gottstein 1984, Lynch et al. 1988, Fong \& Lau 2004). Finally, the lack of reliable immunological tests to diagnose this disease may underestimate the real prevalence in the population.

In this current work, we evaluated the usefulness of semi-purified antigens obtained from adult $T$. canis extracts as potential immunodiagnostic antigens. Although the adult worms are not responsible for human toxocariasis, their antigens are highly specific and sensitive for toxocariasis serodiagnosis, probably due to the conserved epitopes expressed during different stages of the parasite. Furthermore, compared to juveniles, adult worms may be a better source of antigens because they are relatively easier to obtain and handle. Previous studies have shown that the ELISA immunodiagnosis of toxocariasis using the crude larval extract of $T$. canis presented several nonspecific reactions (Camargo et al. 1992). Similarly, the reduced specificity and high cross-reactivity with other parasites were also observed in the present work when we used the crude extracts from adult worms. To address this issue, several authors have demonstrated that using recombinant antigens from larval $T$. canis increases the specificity of ELISA immunodiagnostic tests (Yamasaki et al. 2000, Fong \& Lau 2004). Therefore, we assessed the specificity and usefulness of different semi-purified antigens from adult Toxocara worm extracts in the serodiagnosis of human toxocariasis.

Given the close taxonomic relationship between $A s$ caris sp. and Toxocara sp., we expected that the sera from Ascaris-infected patients would cross-react in a serological diagnosis because the two ascarids undoubtedly share antigens and epitopes (Watthanakulpanich et al. 2008). Indeed, cross-reactions with Ascaris sp. have already been extensively described in the literature (Nicholas et al. 1986, Camargo et al. 1992). Therefore, to diminish possible cross-reactions, we used sera that had been absorbed with $A$. sum antigens (de Savigny et al. 1979, Nicholas et al. 1986, Roldán et al. 2006) prior to performing the assays with Toxocara sp. semi-purified antigens. Although the sera of patients infected with $A s-$ caris lumbricoides were not examined in this study, we minimized the possibility that the semi-purified antigens would cross-react with samples positive for ascariasis.

An antigen is considered to be specific for an ELISA diagnosis when a minimum specificity of $90 \%$ is demonstrated (Glickman et al. 1978). Notably, most of the semi-purified antigens we tested had specificity values below this cut-off value. However, the specificity may not be a major concern in developed countries with temperate climates, where parasite co-infections are uncommon and infections with soil-transmitted helminths are less prevalent. Conversely, a low specificity may be a significant problem for the serodiagnosis of disease in the tropics, where people might be co-infected with different parasites (Noordin et al. 2005). In our study, the $42,58,68$ and $97-\mathrm{kDa}$ antigens showed high specificity and sensitivity values for both IgM and IgG antibodies at the dilution of 1:40 and were used to further examine the cross-reactivity with patients presenting neurocysticercosis, schistosomiasis or Chagas disease. The 42 and 97$\mathrm{kDa}$ antigens significantly cross-reacted with other par- 

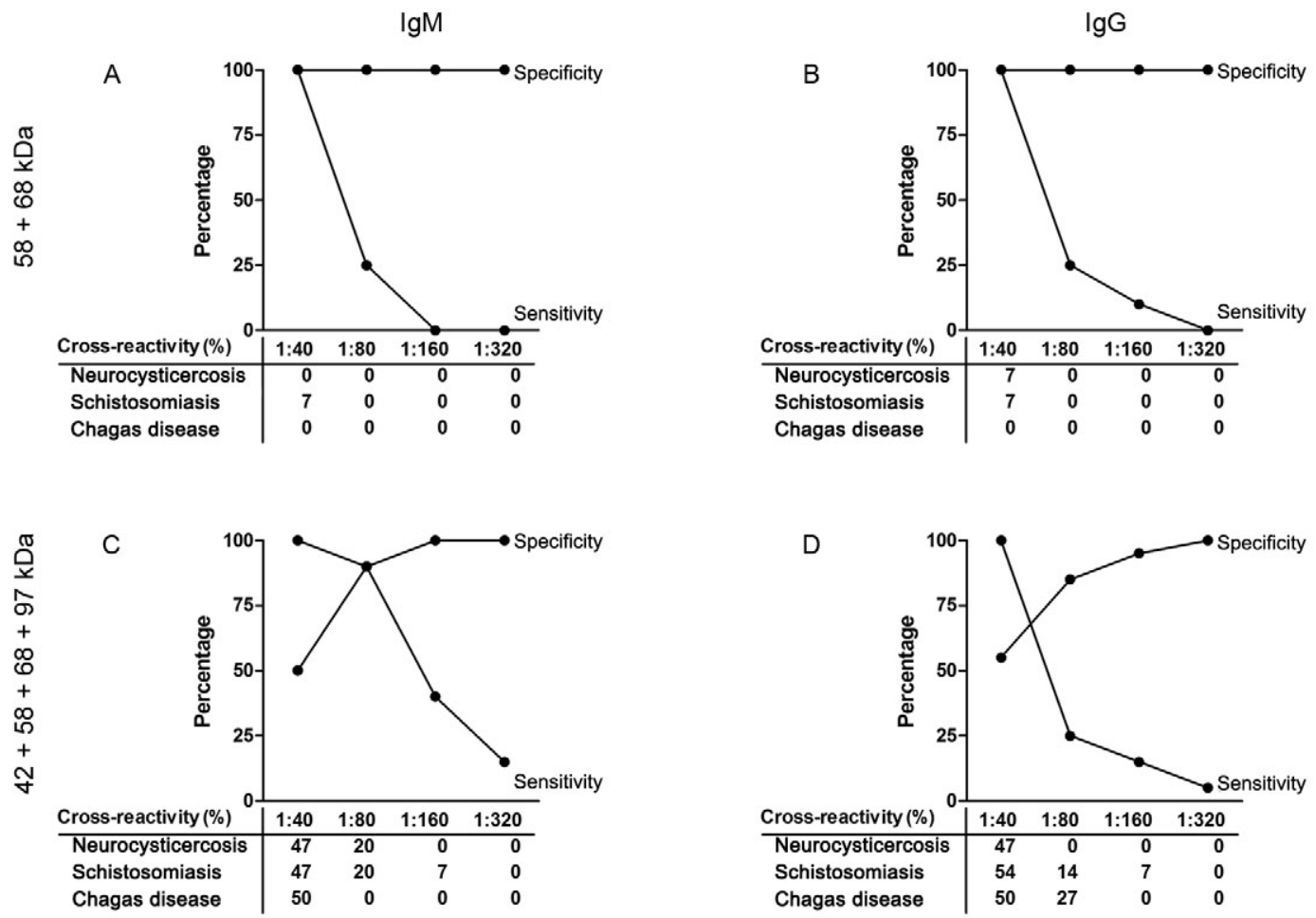

Fig. 4: sensitivity, specificity and cross-reactivity of two combinations of semi-purified Toxocara canis antigens at different serum dilutions. Enzime linked immunosorbent assays were performed using both $\operatorname{IgM}(\mathrm{A}, \mathrm{C})$ or $\operatorname{IgG}(\mathrm{B}, \mathrm{D})$ isotypes. Percentage of serum cross-reactivity against different parasitic infections is shown on the table below each figure.

asites at the 1:40 and 1:80 dilutions. However, the 58 and $68-\mathrm{kDa}$ antigens minimally cross-reacted with all titres of the IgM antibodies from patients with other parasitic infections, although these antigens significantly crossreacted with the IgG antibodies at the titre of 1:40. Taken together, these data suggested that the 58 and $68-\mathrm{kDa}$ antigens might be suitable for the immunodiagnosis of both acute (using IgM at any serum dilution) and chronic (using $\operatorname{IgG}$ at serum dilutions higher than 1:40) phases of human toxocariasis. It is important to note that the crude extract consists of mostly soluble cytosolic proteins with no membrane proteins; the methodology used for the crude extract preparation almost certainly leaves the cellular organelles intact.

When the combination of all four antigens $(42 \mathrm{kDa}$, $58 \mathrm{kDa}, 68 \mathrm{kDa}$ and $97 \mathrm{kDa}$ ) was tested, the specificity values were significantly reduced at lower dilutions (1:40 and 1:80). However, the combination of the 58 and $68-\mathrm{kDa}$ antigens showed the highest specificity and sensitivity at the 1:40 titre (for both IgG and IgM antibodies) and also showed reduced cross-reactivity with other parasites (lower than 10\%). This result thus indicated that the combination of the 58 and $68-\mathrm{kDa}$ antigens was specific and sensitive for the anti-Toxocara antibody, supporting the possibility of using these two antigens for the immunodiagnosis of human toxocariasis in lowprevalent areas. Of course, the parameters chosen for the ELISA assay (e.g., serum dilution, antibody isotype etc.) should take into account the background seroprevalence in the target population.
In conclusion, this study highlights the shortcoming of the Toxocara adult IgG and IgM-ELISA serodiagnosis. The 58 and $68-\mathrm{kDa}$ antigens, either alone or in combination, was shown to be the most promising candidates for the serodiagnosis of human toxocariasis. The limited cross-reactivity and the relatively simple process for obtaining these antigens indicated that they could also be used in areas where other parasitic infections are highly endemic. The use of these antigens in a sequential order might improve the reliability of the diagnosis. In the current study, we evaluated the cross-reactivity against a limited number of parasitic infections. Thus, further tests need to be performed to test whether these semi-purified adult Toxocara antigens might cross-react with other parasitic infections, mainly those caused by nematodes, to further confirm the specificity of such antigens. Moreover, it is important to establish whether the semi-purified adult antigens present similar efficacy to those presented by the larval ES antigen, which is currently used as a standard diagnostic reagent. Finally, the cloning, expression and production of these promising antigens as recombinant antigens will require further research. Such an approach would not only improve the standardization of routine diagnoses, but also allow the determination of the epidemiology of human toxocariasis through seroprevalence surveys.

\section{ACKNOWLEDGEMENTS}

To Dr Fausto G Araujo, for providing parasite negative serum samples from Palo Alto Medical Foundation, Palo Alto, USA. 


\section{REFERENCES}

Buck AA, Gart JJ 1966. Comparison of a screening test and a reference test in epidemiologic studies. I. Indices of agreement and their relation to prevalence. Am J Epidemiol 83: 586-592.

Camargo ED, Nakamura PM, Vaz AJ, da Silva MV, Chieffi PP, de Melo EO 1992. Standardization of dot-ELISA for the serological diagnosis of toxocariasis and comparison of the assay with ELISA. Rev Inst Med Trop Sao Paulo 34: 55-60.

Chiodo P, Basualdo J, Ciarmela L, Pezzani B, Apezteguía M, Minvielle M 2006. Related factors to human toxocariasis in a rural community of Argentina. Mem Inst Oswaldo Cruz 101: 397-400.

De Andrade Lima Coelho R, De Carvalho LB Jr, Perez EP, Araki K, Takeuchi T, Ito A, Aoki T, Yamasaki H 2005. Prevalence of toxocariasis in northeastern Brazil based on serology using recombinant Toxocara canis antigen. Am J Trop Med Hyg 72: 103-107.

de Savigny DH, Voller A, Woodruff AW 1979. Toxocariasis: serological diagnosis by enzyme immunoassay. J Clin Pathol 32: 284-288.

Despommier D 2003. Toxocariasis: clinical aspects, epidemiology, medical ecology and molecular aspects. Clin Microbiol Rev 16: 265-272.

Espinoza YA, Huapaya PH, Roldán WH, Jiménez S, Arce Z, Lopez E 2008. Clinical and serological evidence of Toxocara infection in school children from Morrope district, Lambayeque, Peru. Rev Inst Med Trop Sao Paulo 50: 101-105.

Fong MY, Lau YL 2004. Recombinant expression of the larval excretory-secretory antigen TES-120 of Toxocara canis in the methylotrophic yeast Pichia pastoris. Parasitol Res 92: 173-176.

Gawor J, Borecka A, Zarnowska H, Marczyńska M, Dobosz S 2008. Environmental and personal risk factors for toxocariasis in children with diagnosed disease in urban and rural areas of central Poland. Vet Parasitol 155: 217-222.

Giacometti A, Cirioni O, Fortuna M, Osimani P, Antonicelli L, Del Prete MS, Riva A, D’Errico MM, Petrelli E, Scalise G 2000. Environmental and serological evidence for the presence of toxocariasis in the urban area of Ancona, Italy. Eur J Epidemiol 16: 1023-1026.

Gillespie SH 1988. The epidemiology of Toxocara canis. Parasitol Today 4: 180-182.

Gillespie SH, Bidwell D, Voller A, Robertson BD, Maizels RM 1993. Diagnosis of human toxocariasis by antigen capture enzyme linked immunosorbent assay. J Clin Pathol 46: 551-554.

Glickman L, Schantz P, Dombroske R, Cypess R 1978. Evaluation of serodiagnostic tests for visceral larva migrans. Am J Trop Med Hyg 27: 492-498.

Glickman LT, Schantz PM 1981. Epidemiology and pathogenesis of zoonotic toxocariasis. Epidemiol Rev 3: 230-250.

Habluetzel A, Traldi G, Ruggieri S, Attili AR, Scuppa P, Marchetti R, Menghini G, Esposito F 2003. An estimation of Toxocara canis prevalence in dogs, environmental egg contamination and risk of human infection in the Marche region of Italy. Vet Parasitol 113: 243-252.

Hotez PJ, Wilkins PP 2009. Toxocariasis: America's most common neglected infection of poverty and a helminthiasis of global importance? PLoS Negl Trop Dis 3: e400.

Iudici Neto F, Pianetti-Filho G, Araújo RN, Nascimento E 2007. Immunodiagnosis of human neurocysticercosis by using semi-purified scolex antigens from Taenia solium cysticerci. Rev Soc Bras Med Trop 40: 163-169.

Jacob CM, Pastorino AC, Peres BA, Mello EO, Okay Y, Oselka GW 1994. Clinical and laboratorial features of visceral toxocariasis in infancy. Rev Inst Med Trop Sao Paulo 36: 19-26.
Jacquier P, Gottstein B, Stingelin Y, Eckert J 1991. Immunodiagnosis of toxocariasis in humans: evaluation of a new enzyme-linked immunosorbent assay kit. J Clin Microbiol 29: 1831-1835.

Jones JL, Kruszon-Moran D, Won K, Wilson M, Schantz PM 2008. Toxoplasma gondii and Toxocara spp co-infection. Am J Trop Med Hyg 78: 35-39.

Jones WE, Schantz PM, Foreman K, Smith LK, Witte EJ, Schooley DE, Juranek DD 1980. Human toxocariasis in a rural community. Am J Dis Child 134: 967-969.

Lowry OH, Rosebrough NJ, Farr AL, Randall RJ 1951. Protein measurement with the Folin phenol reagent. J Biol Chem 193: 265-275.

Lynch NR, Wilkes LK, Hodgen AN, Turner KJ 1988. Specificity of Toxocara ELISA in tropical populations. Parasite Immunol 10: 323-337.

Magnaval JF, Fabre R, Maurieres P, Charlet JP, de Larrard B 1991. Application of the western blotting procedure for the immunodiagnosis of humantoxocariasis. Parasitol Res 77: 697-702.

Magnaval JF, Glickman LT, Dorchies P, Morassin B 2001. Highlights of human toxocariasis. Korean J Parasitol 39: 1-11.

Marmor M, Glickman L, Shofer F, Faich LA, Rosenberg C, Cornblatt B, Friedman S 1987. Toxocara canis infection of children: epidemiologic and neuropsychologic findings. Am J Public Health 77: 554-559.

Nicholas WL, Stewart AC, Walker JC 1986. Toxocariasis: a serological survey of blood donors in the Australian Capital Territory together with observations on the risks of infection. Trans $R$ Soc Trop Med Hyg 80: 217-221.

Noordin R, Smith HV, Mohamad S, Maizels RM, Fong MY 2005. Comparison of IgG-ELISA and IgG4-ELISA for Toxocara serodiagnosis. Acta Trop 93: 57-62.

Roldán W, Cornejo W, Espinoza Y 2006. Evaluation of the dot enzyme-linked immunosorbent assay in comparison with standard ELISA for the immunodiagnosis of human toxocariasis. Mem Inst Oswaldo Cruz 101: 71-74.

Schantz PM 1989. Toxocara larva migrans now. Am J Trop Med Hyg 41 (Suppl. 3): 21-34.

Schantz PM, Glickman LT 1983. Ascarids of cats and dogs: a public health and veterinary medicine problem. Bol Oficina Sanit Panam 94: 571-586.

Smith H, Holland C, Taylor M, Magnaval JF, Schantz P, Maizels R 2009. How common is human toxocariasis? Towards standardizing our knowledge. Trends Parasitol 25: 182-188.

Smith HV, Noordin R 2006. Diagnostic limitations and future trends in the serodiagnosis of human toxocariasis. In Holland CV, Smith HV (eds.), Toxocara: the enigmatic parasite, CABI Publishing, Oxfordshire, p. 89-112.

Speiser F, Gottstein B 1984. A collaborative study on larval excretory/ secretory antigens of Toxocara canis for the immunodiagnosis of human toxocariasis with ELISA. Acta Trop 41: 361-372.

Taylor MR, Keane CT, O'Connor P, Mulvihill E, Holland C 1988. The expanded spectrum of toxocaral disease. Lancet 1: 692-695.

Watthanakulpanich D, Smith HV, Hobbs G, Whalley AJ, Billington D 2008. Application of Toxocara canis excretory-secretory antigens and IgG subclass antibodies (IgG1-4) in serodiagnostic assays of human toxocariasis. Acta Trop 106: 90-95.

Wolfe A, Wright IP 2003. Human toxocariasis and direct contact with dogs. Vet Rec 152: 419-422.

Yamasaki H, Araki K, Lim PK, Zasmy N, Mak JW, Taib R, Aoki T 2000. Development of a highly specific recombinant Toxocara canis second-stage larva excretory-secretory antigen for immunodiagnosis of human toxocariasis. J Clin Microbiol 38: 1409-1413. 